\title{
Basal Cell Carcinoma Developed on an Active Lesion of Mucocutaneous Leishmaniasis: A Case Report from Yemen
}

Mohamed A. Al-Kamel, MD, FISD (USA)*

\author{
' Regional Leishmaniasis Control Center (RLCC), Sana'a, Yemen \\ 2 Department of Dermatology, Sana'a University Hospital, Sana'a, Yemen
}

\begin{abstract}
Cutaneous leishmaniasis (CL) and basal cell carcinoma (BCC) are two common clinical entities in Yemen. The hypothesis of concurrent occurrence of leishmaniasis and malignancy has been reported from several regions worldwide, and in many of them, leishmaniasis has been indicated as a risk factor for the development of malignant lesions with a direct or/and indirect involvement. Hereby, the author reports a case of BCC (Clark level IV) that had been developed on an active lesion of mucocutaneous leishmaniasis (MCL), supposing that carcinogenesis in the current case was triggered by Leishmania parasites and influenced by sun's ultraviolet (UV) radiation exposure. Further studies with appropriate methods and experimental approaches are recommended to carefully address these challenging hypotheses.
\end{abstract}

\section{Introduction}

Cutaneous leishmaniasis (CL), a chronic granulomatous inflammation caused by an infection of intracellular Leishmania parasite, is a worldwide challenging disease with significant endemicity in Yemen. Mucocutaneous leishmaniasis (MCL) is the most prevalent form of $\mathrm{CL}$ in Central Yemen, where the rural population is predominantly affected, particularly the children and the women. Leishmania major is referred to as the principal causative agent in these cases [1].

Basal cell carcinoma $(B C C)$ is the most common histological type of skin cancer that prevails in Yemen, where malignant skin tumors account for $10 \%$ of all malignancies. Its prevalence is high among the elderly and the most common lesion sites are on the sun-exposed skin [2,3].

$\mathrm{BCC}$ rarely metastasizes; however, it could spread into nearby tissues and organs. Development of BCC is strongly associated with several risk factors, such as family history, intense ultraviolet exposure, immune suppression, chronic irritation, and chronic skin ulcers [4].

Furthermore, recent studies has indicated that $\mathrm{CL}$ may act as a risk factor for the development of various cancerous lesions on the skin, including BCC [5]. Hereby, a case of BCC developed on an active lesion of $\mathrm{MCL}$, which had been partially managed at the Regional Leishmaniasis Control Center (RLCC), Yemen.

\section{Case Report}

A 70-year-old male farmer from Raimah (a leishmaniasis endemic governorate at Central Yemen) visited RLCC clinic in Sana'a with three ulcerated lesions on his nose, the lesions was presented for at least seven months. Clinical examination revealed three scaling, shiny, ulcerated, red, and pearly nodules (5-10 $\mathrm{mm}$ in diameter) on the left alar rim (Figers. $1 \mathrm{~A}$ and 1B).

History of the present illness revealed that over three years ago, the patient had an ulcer with diffuse erythema on the top of his nose, which was diagnosed by microscopic examination of a slit skin smear as $\mathrm{MCL}$ with a heavy infection of Leishmania parasites, and he was treated with intramuscular and intralesional injections of Sodium Stibogluconate (SSG). Although, it was responding well to SSG therapy, another concurrent pearly well-circumscribed ulcer with raised areas and ulcerated center started to develop at the site of the regressing leishmanial lesion.
Gradually it deeply invaded the nearby tissue and caused a significant destruction of the nose. BCC was diagnosed through histopathologic examination of a biopsied specimen. Accordingly, the cancer was excised by a plastic surgeon without post-operative radio- or chemotherapy. The patient was left with an unpleasant permanent disfigurement of the nose. (Figure 1B).

Seven months postoperative, three tumorous lesions had redeveloped on the left aral rim (Figers 1A and 1B), the lesions were biopsied and histopathologically identified as recurrent nodular BCC of the nose (Clark level IV); histopathologic study showed an ulcerated tumor growth composed of atypical basaloid cells arranged in cohesive nets with peripheral palisading and retraction artifact. Furthermore, the patient reported a history of prolonged sun exposure and a relevant family history of cutaneous leishmaniasis.

At RLCC, the patient had undergone two cryotherapy sessions with liquid nitrogen with two weeks interval (Figure 2). A complete clearance of the malignant lesions was obtained within one month of follow-up.

\section{Discussion and Conclusion}

The extrinsic inflammation in cancers is caused by many factors, such as microbial infection, ultraviolet (UV) light radiation, autoimmune diseases, obesity, smoking, and irradiation, although by far the UV radiation is the most important factor in the malignant lesion development and progression. In contrast, cancer-intrinsic inflammation can be triggered by cancer-initiating mutations and can contribute to malignant progression through the recruitment and activation of inflammatory cells. Both extrinsic and intrinsic inflammation can result in immunosuppression, thereby providing a preferred background for tumor development $[6,7]$.

Basal cell carcinoma (BCC), an epithelial malignant tumor with a low malignant potential, may arise in the setting of scars, draining sinuses, ulcers, burn sites, and foci of chronic inflammation. There is no unified and generally accepted classification of BCCs. When classifying BCCs, most authors start from the growth pattern, which gives more information about the bio-behavior and less often from the differentiation of tumors. All published works are in accord regarding their determination of the three basic groups of BCCs: nodular, superficial, and infiltrative, which present $80-90 \%$ of all BCCs. The diagnostic histological features common for all types of BCC are basaloid cells with a thin pale cytoplasm 


\section{CASE REPORT}

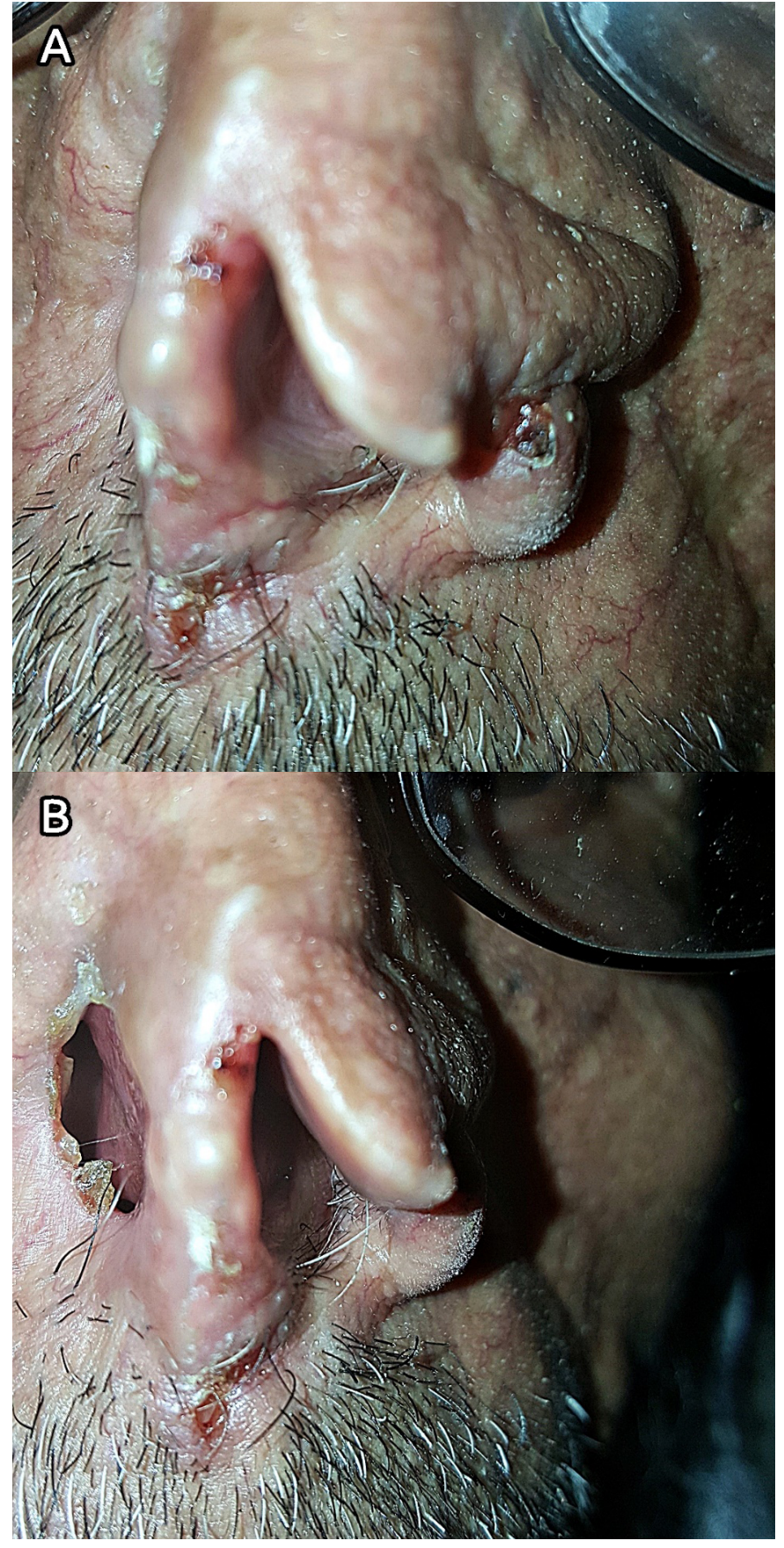

Figure 1. (A) Lesions on the rim of the left nostril (close view). (B) Post-operative disfigurement of the nose.

surrounding round or oval nuclei with a rough granulated chromatin pattern. The peripheral borderline cell layers are characterised by palisade arrangement and the surrounding stroma is often separated by artificially created slits, whereas the internal arrangement of the cells is rather chaotic. Most tumors originate in the epidermis and invade the dermis in the form of solid or cystic nodules or streaky projections creating various growth patterns $[7,8]$.

In the literature, there are many cases of BCC developed during or after leishmaniasis on the skin $[4,9]$. The first article discussing $C L$ as a possible predisposing factor for skin malignancy in Yemen was reported by Morsy et al. who described that the histopathological findings of a disfiguring $\mathrm{CL}$ lesion showed a picture as typical as of BCC [10].

In the previous studies, four possibilities were described to define the concurrent presence of BCC and CL in humans: (1) leishmaniasis masquerading as a malignant disorder; (2) leishmaniasis developing as

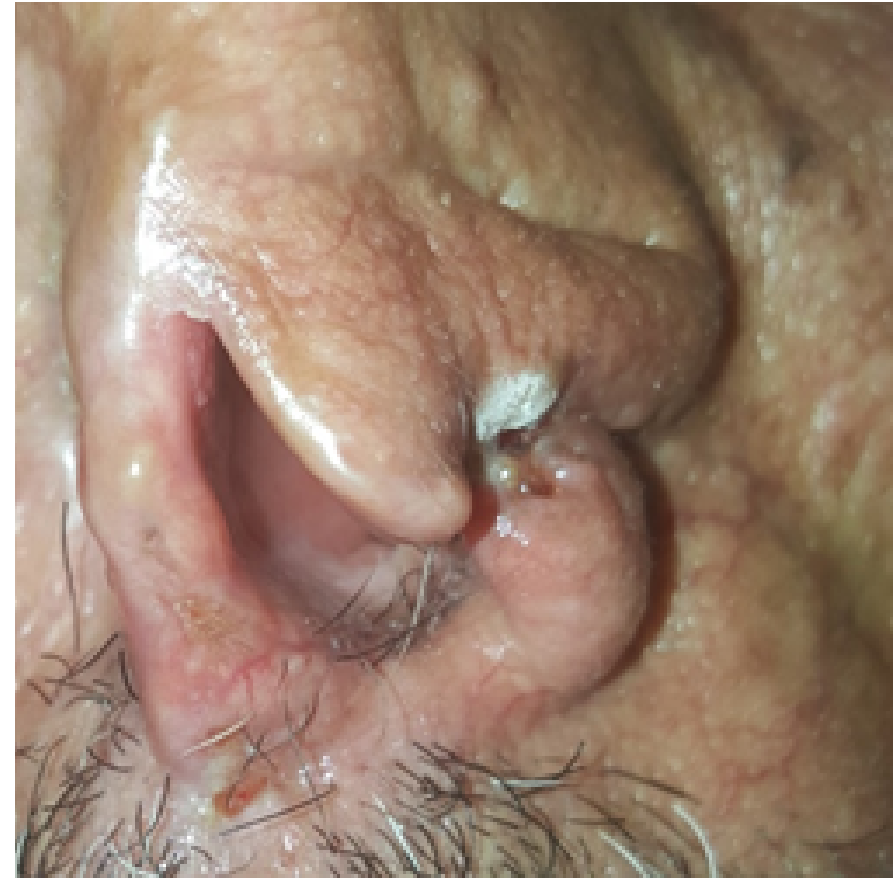

Figure 2. Cancerous lesions two weeks after the first cryotherapy session.

a difficult to diagnose and treat infection among patients receiving chemotherapy for various malignant disorders; (3) simultaneous diagnosis of leishmaniasis and a neoplastic disorder in the same tissue samples of immunocompromised patients; and (4) direct involvement of Leishmania species in the pathogenesis of cancerous lesions [5].

Carcinogenic pathways in the current case of nodular, recurrent BCC (Clark level IV) of the nose may include, chronic inflammation induced by Leishmania parasites; chronic irritation induced by prolonged sun exposure (UV radiation); and subsequent dysfunction of the immune system $[5,11]$ Further studies with appropriate methods and experimental approaches are recommended to carefully address these challenging hypotheses.

\section{Article Information \\ *Correspondence: Mohamed Ahmed Al-Kamel, MD, FISD(USA)}

Founding Chair, Regional Leishmaniasis Control Center (RLCC), Taiz St., Sana'a, P.O. Box 12692, Yemen.

E-mail: dralkamel@hotmail.com

Received: Jul. 26, 2017; Accepted: Jun. 28, 2018; Published: Jul. 30, 2018

DOI: $10.24983 /$ scitemed.acd.2018.00072

Copyright $\odot 2018$ The Author (s). This is an open-access article distributed under the terms of the Creative Commons Attribution 4.0 International License (CC-BY).

\section{Funding: None}

\section{Conflict of Interest: None}

Informed Consent: Written patient informed consent was obtained.

\section{Keywords}

Basal cell carcinoma; malignancy; mucocutaneous leishmaniasis; neoplasia; Yemen. 


\section{References}

1. Al-Kamel MA. Leishmaniasis in Yemen: a clinicoepidemiological study of leishmaniasis in central Yemen. Int J Dermatol 2016;55:849855.

2. AlZou AB, Thabit MA, AlSakkaf KA, Basaleem HO. Skin Cancer: ClinicoPathological Study of 204 Patients in Southern Governorates of Yemen. Asian Pac J Cancer Prev 2016;17(7):3195-3199.

3. Forae GD, Olu-Eddo AN. Malignant skin tumors in benin city, SouthSouth, Nigeria. Oman Med J 2013;28(5):311-315.

4. Chisti M, Almasri R, Hamadah I. Is cutaneous leishmaniasis a risk factor for basal cell carcinoma? Gulf J Oncolog 2016;1(21):64-66.

5. Kopterides P, Mourtzoukou EG, Skopelitis E, Tsavaris N, Falagas ME. Aspects of the association between leishmaniasis and malignant disorders. Trans R Soc Trop Med Hyg 2007;101(12):1181-1189.
6. Todoric J, Antonucci L, Karin M. Targeting inflammation in cancer prevention and therapy. Cancer Prev Res (Phila) 2016;9(12):895-905.

7. Crowson AN. Basal cell carcinoma: biology, morphology and clinical implications. Mod Pathol 2006;19:S127-S147.

8. Vantuchová Y, Čuřík R. Histological types of basal cell carcinoma. Scr Med (Brno) 2006;79:261-270.

9. Al-Kamel MA. Cutaneous T Cell lymphoma (CTCL) superimposed on disseminated cutaneous leishmaniasis (DCL) in an immunocompromised female from Yemen. International Journal of Clinical Dermatology \& Research 2017;:S3:002:4-8.

10. Morsy TA, Mangoud AM, el-Sebai MM, al Seghayer SM. Cutaneous leishmaniasis as a possible predisposing factor for skin malignancy. J Egypt Soc Parasitol 1992;22(3):599-602.

11. Al-Kamel MA. Leishmaniasis and malignancy: a. review and perspective. Clinical Skin Cancer 2017;2(1-2):54-58. 